\title{
Nail apparatus melanoma initially diagnosed as nail matrix blue nevus: a case report with dermatoscopy and dermatopathology
}

\author{
Bengu Nisa Akay ${ }^{1}$, Aylin Okcu Heper ${ }^{2}$, Luc Thomas ${ }^{3}$, Brigitte Balme ${ }^{3}$, Simon Clark ${ }^{4,5}$, \\ Cliff Rosendahl ${ }^{5,6}$
}

\begin{abstract}
1 Department of Dermatology, Ankara University Faculty of Medicine, Ankara, Turkey
2 Department of Pathology, Ankara University Faculty of Medicine, Ankara, Turkey

3 Dermatology and Dermatopathology Centre, Hospitalier Lyon Sud, Pierre Bénite, France

4 DHM Pathology, Macquarie Park, NSW, Australia

5 Tehran University of Medical Sciences, Tehran, Iran

6 School of Medicine, The University of Queensland, Australia
\end{abstract}

Key words: nail, melanoma, blue nevus, dermatoscopy

Citation: Akay BN, Heper AO, Thomas L, Balme B, Clark S, Rosendahl C. Nail apparatus melanoma initially diagnosed as nail matrix blue nevus: a case report with dermatoscopy and dermatopathology. Dermatol Pract Concept. 2017;7(1):13. DOI: https://doi.org/10.5826/ dpc.0701a13

Received: June 27, 2016; Accepted: October 22, 2016; Published: January 31, 2017

Copyright: @2017 Akay et al. This is an open-access article distributed under the terms of the Creative Commons Attribution License, which permits unrestricted use, distribution, and reproduction in any medium, provided the original author and source are credited.

Funding: None.

Competing interests: The authors have no conflicts of interest to disclose.

All authors have contributed significantly to this publication.

Corresponding author: Bengu Nisa Akay, MD, Ankara Universitesi Tip Fakultesi, Ibni Sina Hastanesi, Dermatoloji Anabilim Dali, 06100 , Altindag, Ankara, Turkey. Email: nisaakay15@yahoo.com

ABSTRACT We present a case of nail apparatus melanoma in a 50-year-old woman presenting as new and changing longitudinal melanonychia of the right thumb. Very heavy melanin pigmentation involving both the epidermis and dermis interfered with dermatopathological assessment, which initially leads to a diagnosis of nail matrix blue nevus. After consultation with a specialist multidisciplinary clinic the diagnosis was revised to invasive melanoma, a diagnosis consistent with the clinical and dermatoscopic assessment.

\section{Case Report}

A 50-year-old woman presented to a dermatology clinic with a pigmented stripe on her right thumb. She reported that the stripe had appeared three years previously and that it had progressively widened. Examination revealed heavily pigmented longitudinal melanonychia (Figure 1a). Dermatoscopy revealed melanonychia, color blue, mainly comprised of structureless blue but with lines parallel on one side. Taking this variability into account, there were lines parallel varying in width but not varying in interval or color (Figure 1b). There was no pigmentation of the proximal nail fold (Hutchinson's sign) or cuticle (micro-Hutchinson's sign), although pigment was visible on the nail plate through the translucent cuticle (pseudo-Hutchinson's sign).

Nail matrix biopsy was performed involving avulsion of the nail plate and longitudinal excision of the entire pigmented portion of the matrix, which was submitted for histological 

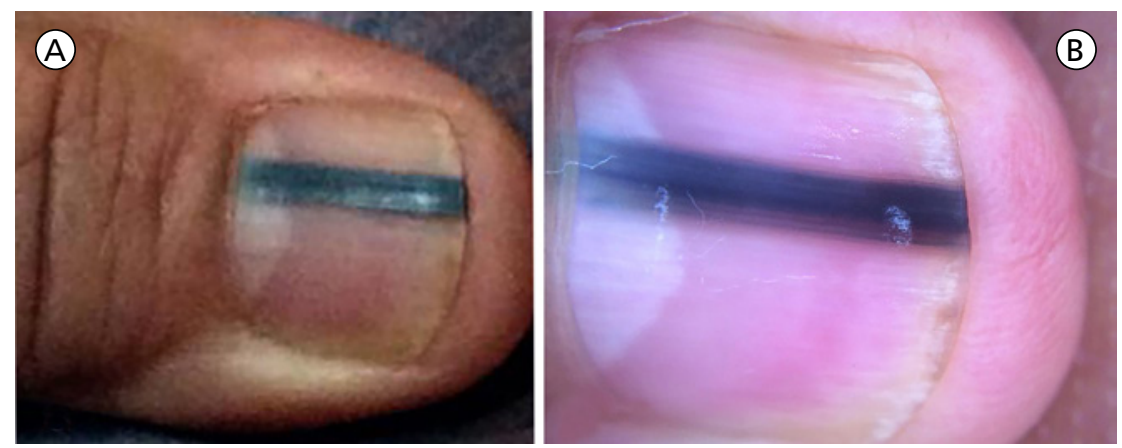

Figure 1. Clinical (a) image of the right thumbnail of a 50-year-old woman. The pigmented stripe has appeared and progressed over three years. Dermatoscopy (b) displays melanonychia striata with lines parallel varying in width, but not interval or color. [Copyright: (C)2017 Akay et al.]

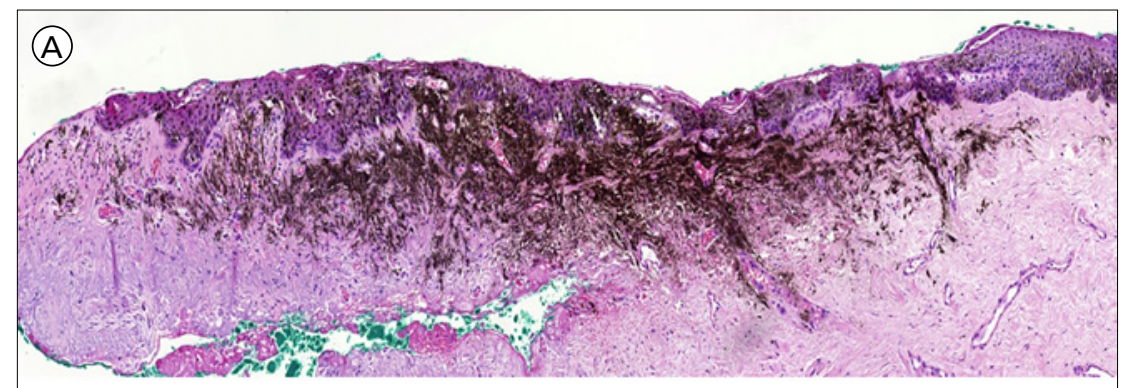

(B)

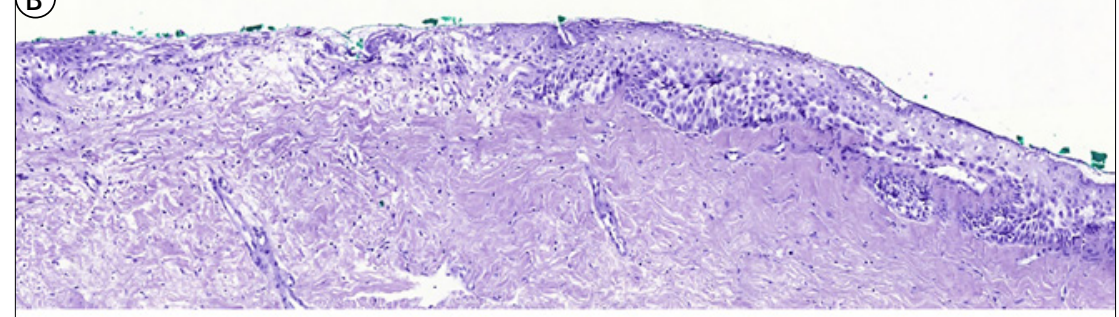

(C)

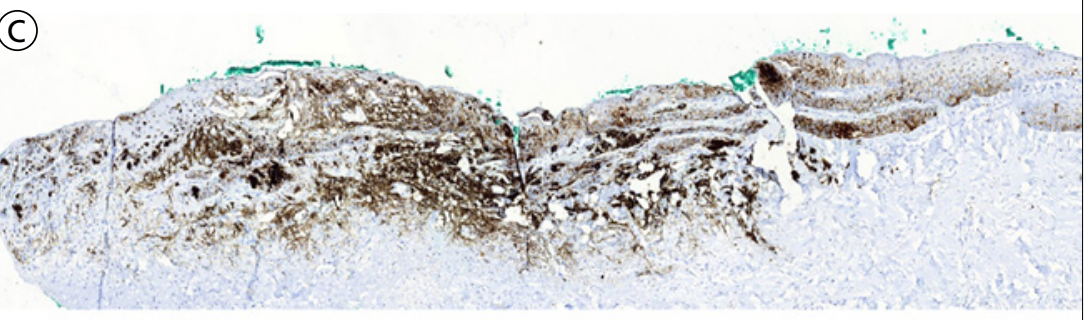

(D)

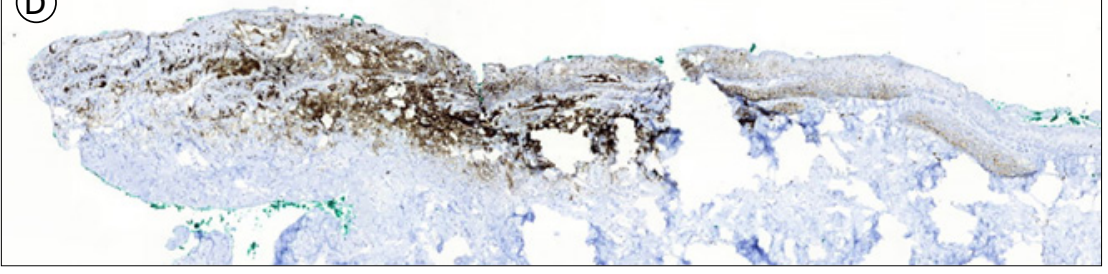

Figure 2. H\&E staining (a) of the matrix biopsy specimen from the lesion in Figure 1 displays heavy pigmentation over both the epidermis and dermis which compromised assessment of architecture and cytology. A bleached section (b) revealed an apparent epidermal melanocytic proliferation, but this was initially interpreted as keratinocytes modified by bleaching. Interpretation of sections stained with Melan A (c) and Ki-67 (d) was compromised by heavy melanin pigmentation substantially obscuring DAB chromagen. [Copyright: (C2017 Akay et al.]

examination by a dermatopathologist. Hematoxylin and eosin (H\&E) stained material (Figure 2a) revealed heavy mel- anin staining throughout the epidermis and into the reticular dermis to such a degree that assessment of the archi- tecture and cytology of the lesion were compromised. Bleached sections were examined (Figure $2 \mathrm{~b}$ ), and although these did reveal an apparent melanocytic proliferation in the epidermis it was considered that this could in fact be artifactual due to the bleaching. An apparent absence of atypia in the dermal spindle cells was considered to be consistent with blue nevus, and in that context the epidermal changes were interpreted as being due to keratinocytes modified by the bleaching process. Sections stained with Melan A (Figure 2c), Ki-67 (Figure 2d) and HMB45 showed staining throughout the epidermis and papillary dermis, but the presence of heavy pigmentation substantially obscured DAB chromogen, making the interpretation difficult and unreliable.

The diagnosis of blue nevus was questioned by the treating dermatologist because the history of a new and continuously growing nail matrix melanocytic lesion at mature age was inconsistent with a benign diagnosis and because abundant epidermal melanin is not expected in a blue nevus.

Clinical, dermatoscopic and dermatopathologic images were reviewed by a multidisciplinary tumor board (including dermatologists and dermatopathologists), and dermatopathology slides were also reviewed by a member of that board (author BB). They rendered a unanimous opinion that this lesion represented an invasive melanoma based on the history of recent onset and progression at mature age, the nature of the dermatoscopic melanonychia striata and the dermatopathological architecture and cytology. They commented that although it was unusual to have such a thick tumor with such a narrow band of melanonychia, this was not inconsistent with the diagnosis.

Following this the dermatopathological diagnosis was revised by the reporting pathologist to that of invasive nail apparatus melanoma, Clark level 2, Breslow thickness $0.38 \mathrm{~mm}$ with focal epidermal erosion and with no 
mitoses or lymphovascular or peri-neural invasion observed. The patient agreed to definitive treatment by distal phalanx amputation.

\section{Conclusions}

Nail apparatus melanoma is uncommon but has a relatively high mortality [1]. There is a reported female preponderance with location on the thumb having the highest prevalence (41\%) [2]. The proportion of nail apparatus melanoma which are pigmented has been reported as $71.7 \%$ [3] with the median Breslow thickness of nail apparatus melanoma being reported as $0.8 \mathrm{~mm}$ with up to $18 \%$ having spindle shaped cells [4]. All reported nail apparatus melanomas have apparently arisen de novo, there being no reports of any pre-existing associated nevus [4]. The diagnosis can be challenging both clinically and dermatopathologically [5], although this is not expected in a mature invasive melanoma. A history of new and changing longitudinal melanonychia at mature age is a clue to malignancy as is the specific dermatoscopic clue to nail apparatus melanoma of longitudinal melanonychia (brown, black, grey or blue) with lines parallel varying in width, interval and color [6]. In the present case, there was one broad band of blue color and two narrow bands of the same color. Lines varying in width, but not interval and color, raised the possibility of a benign etiology, but this appearance could also be explained by the very heavy density of pigmentation. In the present case, the index of suspicion of the treating dermatologist was high and the patient was referred promptly for nail matrix biopsy. Subsequent dermatopathological assessment, including H\&E, Melan A and Ki-67 staining, was hindered by dense melanin deposition. Bleaching of histological sections was employed in response to this, but it is known that this process can damage the tissue, compromising interpretation [7]. As a result of the dense melanin deposition and equivocal dermal melanocyte cytology, an initial dermatopathological diagnosis of blue nevus was rendered.

Blue nevi are expected to appear on the skin at mature age, but by the time they are observed they are generally stable [8]. Blue nevus has rarely been reported in the nail apparatus [9-18], and although two reported cases presented with longitudinal melanonychia $[16,17]$, the others all presented with structureless subungual pigmentation (one had associated periungal pigmentation [18]), as might be expected in a dermal pigmented melanocytic proliferation.

This diagnosis of blue nevus was questioned primarily because of a history of progressive evolution clinically and also because, with only two exceptions in the literature, nail apparatus blue nevus does not exhibit epidermal pigmentation or longitudinal melanonychia. A pigmented melanocytic proliferation restricted to the dermis of the nail matrix is not expected to transfer pigment to the developing nail plate, and in this case the abundance of melanin in the epidermis was evidence contrary to a diagnosis of nail matrix blue nevus.

Clinical and dermatoscopic information can be critical to the correct interpretation of difficult dermatopathological material. The appearance at mature age, and progressive widening of longitudinal melanonychia in the form of lines in the color of melanin, is compelling evidence for melanoma and any alternative report should only be accepted if the dermatopathology is unequivocal. In the presented case, heavy pigmentation complicated dermatopathological assessment, but despite this, the final revised signed-out diagnosis, facilitated by consultation with a tertiary multidisciplinary clinic, conformed to the clinical and dermatoscopic assessment.

\section{References}

1. Banfield CC, Redburn JC, Dawber RP. The incidence and prognosis of nail apparatus melanoma. A retrospective study of 105 patients in four English regions. Br J Dermatol. 1998;139:276-279.

2. Phan A, Touzet S, Dalle S, Ronger-Savlé S, Balme B, Thomas L. Acral lentiginous melanoma: a clinicoprognostic study of 126 cases. Br J Dermatol. 2006;155:561-569.

3. Phan A, Dalle S, Touzet S, Ronger-Savlé S, Balme B, Thomas L. Dermoscopic features of acral lentiginous melanoma in a large series of 110 cases in a white population. Br J Dermatol. 2010;162:765-771.

4. Phan A, Touzet S, Dalle S, Ronger-Savlé S, Balme B, Thomas L. Acral lentiginous melanoma: histopathological prognostic features of 121 cases. Br J Dermatol. 2007;157:311-318.

5. Weedon D, Van Deurse M, Rosendahl C. 'Occult” melanocytes in nail matrix melanoma. Am J Dermatopathol. 2012;34:855.

6. Thomas L, Dalle S. Dermoscopy provides useful information for the management of mlanonychia striata. Dermatol Ther. 2007;20:3-10. PMID: 17403255 DOI: 10.1111/j.15298019.2007.00106.x

7. Suvarna KS, Layton C, Bancroft JD, eds. Bancroft's Theory and Practice of Histological Techniques. 7th ed. London: Churchill Livingstone; 2012:251.

8. Cabral ES, Chen FW, Egbert BM, Swetter SM. Acquired blue nevi in older individuals: retrospective case series from a Veterans Affairs population, 1991 to 2013. JAMA Dermatol. 2014;150:873876.

9. Causeret AS, Skowron F, Viallard AM, Balme B, Thomas L. Subungual blue nevus. J Am Acad Dermatol. 2003;49: 310-312

10. Dalle S, Ronger-Savle D, Cicale L, Balme B, Thomas L. A bluegray subungual discoloration. Arch Dermatol. 2007;143:937-942

11. Kim HS, Kim YJ, Kim JW, Yu DS. Subungual blue nevus. J Eur Acad Dermatol Venereol. 2006;21:271-272.

12. Moulonguet-Michau I, Abimelec P. Nail unit blue melanocyte nevi: 2 case reports. [French]. Ann Dermatol Venereol. 2004;131:984-986.

13. Salasche SJ, Garland LD. Tumors of the nail. Dermatol Clin. 1985;3:501-519.

14. Smith DF, Morgan MB, Bettencourt MS, Haley JA. Longitudinal melanonychia. Arch Dermatol. 2003;139:1209-1014.

15. Vidal S, Sanz A, Hernández B, Yus ES, Requena L, BaranR. Subungual blue nevus. Br J Dermatol. 1997;137:1023-1025. 
16. Soyer HP, Kerl H. European society of pediatric dermatology clinical case reports 1984. In: Baran R, Dawber RPR, Berker DAR, Haneke E, Tosti A (eds). Diseases of the Nails and Their Management. 3rd ed. Oxford: Blackwell Science Ltd; 2001:616-618.

17. Naylor EMT, Ruben BS, Robeinson-Bostom L, Telang GH,
Jellinek NJ. Subungual blue nevus with combined phenotypic features. J Am Acad Dermatol. 2008;58:1021-1024.

18. Gershtenson PC, Krunic A, Chen H, Konanahalli M, Worobec S. Subungual and periungual congenital blue nevus. Australas J Dermatol. 2009;50:144-147. 